\title{
When Do Regional Research Collaborations Make Sense?
}

\author{
Steven F. Warren
}

Vice Provost for Research \& Graduate Studies, University of Kansas

\begin{abstract}
7 ollaboration has become the drumbeat of science and innovation. This fact reflects the widespread recognition of the power that comes from bringing different knowledge sets and skills to together to solve complex problems. However, it is also widely recognized that collaboration is often difficult and comes at a price. It requires lots of social interaction, trust, and typically needs to have some obvious advantages for all involved parties. In my experience people do not engage in collaboration because it's fun (although sometimes it is) or it is nice to do. They engage in it because it is necessary to solve an important problem.
\end{abstract}

The ability to communicate instantly with virtually anyone in the world has also opened the door to collaborations with virtually anyone. Still, it is usually easier and often more satisfying to collaborate with a colleague whose office is down the hall. Thus local collaborations will continue to be the dominant form as measured by sheer numbers of participants. But if there is a good reason to collaborate with someone across the country or on the other side of the world, scientists and innovators think nothing of it these days. Email, Skype, secure websites, relatively cheap and frequent air travel - all of these changes in the basic infrastructure of communication have greatly lowered the cost of collaborations over great distances. For example, I collaborate on research projects with colleagues at several other universities and many of my colleagues carry on collaborations literally all over the globe. This has become so common that we think little of it.

Given the relative ease of communicating and collaborating with great talent anywhere in the world, why would we want to limit ourselves to "regional collaborations"? In fact, such an idea sounds quaint or provincial and more importantly like a poor strategy for competing successfully in an era of globalization. Nevertheless, regional collaborations may still have their place. Thus, I offer for your consideration three scenarios in which regional collaborations may be exactly the right approach. These are 1) uniquely regional research problems; 2) the development and maintenance of certain types of expensive research infrastructure; and 3) some regional economic development initiatives. 


\section{Uniquely Regional Research Problems}

Some possible topics for regional research collaboration include climate change, energy, and water. Each of these are complex issues is likely to have uniquely regional dimensions. For example, on the Great Plains from Texas north to the Dakotas, water availability and quality is an increasingly critical issue that spans the region. Likewise, the related topic of climate change associated with global warming is likely having some unique regional impacts. Rainfall (already often marginal) is likely to diminish on the western side of the Great Plains while the vast Ogallala Aquifer, the underground sea that runs under the western plains is being rapidly pumped dry in many areas. Thus, both water management and ecological forecasting are good examples of topics for which regional collaboration is potentially critical on many levels. It is not surprising then that a regional climate change research initiative is already underway. This has brought together a consortium of four universities in Kansas and Oklahoma (the University of Kansas, Kansas State University, the University of Oklahoma, and Oklahoma State University). These universities are taking advantage of funding available to them as EPSCoR states by the National Science Foundation to collaborate in the creation of a "cyber commons" - a powerful, integrated cyber environment for knowledge discovery and education across complex environmental phenomena. Specifically, this cyber Commons will integrate two frameworks - the science framework of data, models, analytics, and narratives and the cyber infrastructure framework of hardware, software, and a collaboration and integration environment. Weaving these frameworks together will allow the collaborating scientists at each university to harness the enormous potential of advanced computing for the purpose of dramatically enhancing ecological forecasting across the Great Plains.

Understanding ecological systems and forecasting their responses to global change is one of the great scientific and technological challenges of the $21^{\text {st }}$ century. Addressing this challenge in the context of the Great Plains is critical for the grasslands that are fundamental to the life and economy of the plains. The utter complexity of the forecasting problem makes it a natural project for multi-disciplinary collaboration with a regional focus.

\section{Regional Infrastructure Needs}

Research increasingly involves the manipulation and study of vast amounts of information, and thus requires the infrastructure to support this. The Great Plains Network (GPN) is a consortium of universities in Midwestern states dedicated to insuring the provision of advanced networking technology throughout the region. Members include universities in Arkansas, South Dakota, Kansas, Oklahoma, Missouri, Nebraska and Iowa. These universities work together to insure common access to the internet2 and related computing infrastructure that is vital to $21^{\text {st }}$ century science. This is a classic win-win situation for all collaborating partners, 
including the respective congressional delegations of each state.

Less formal collaborations occur around highly specialized scientific research cores. For example, some molecular biologists at KU send their DNA samples to the genomic analysis core at Iowa State. This is cost-effective for these KU scientists and at the same time supports the provision of expensive specialized equipment and expertise at Iowa State. Likewise KU looks to scientists from other Midwestern universities help it to use its expensive High Throughput Screening Facility. In reality, these resources can be supported by any university or company, but regional support makes for good neighbors. Nevertheless, this type of regional collaboration remains subject to the relentless rules of globalization - if scientists can get the same service better, faster, and/or cheaper outside the region - then that is probably where they will ultimately send their business.

\section{Regional Economic Development}

Certain types of regional economic development may also generate collaboration among scientists, universities, and businesses. For example, the development of high technology economic development initiatives in the greater Boston, San Francisco, and Boston areas have had synergetic effects that have spawned all sorts of opportunities for these regions. Likewise, it can argued that the growth and development of the so-called Animal Health Corridor in Missouri and Kansas has the potential to benefit the biosciences more broadly in general in this region - including biosciences at the University of Kansas - which are focused primarily on human biosciences. These initiatives can bring with them specialized research facilities, talented scientists and innovators, venture capital, and a creative class that can spawn economic growth and development with regional benefits. Indeed, one could argue that any collaborative effort that builds the scientific and technological resources of a region may well lead to increasing collaboration to the benefit of all. Even in a globalized world, regional strengths still matter.

\section{Potential Barriers}

There are at least three barriers to regional collaboration. The first is the political constraints associated with state lines. This fact of life can make certain types of collaboration difficult to pull off. For example, if collaboration means that one state is going to "win more than another", why would folks in neighboring states support this? Why for example would folks in Missouri or Iowa support a major economic development in Kansas? The answer is that they won't if they are also competitive for the same prize. However, if they are not, then it may be advantageous for one state to support the other. A case in point is the political support that many states have given to the moving the National Bio and AgroDefense Facility to Manhattan, Kansas. Much of the direct benefit of this effort may be localized in Kansas. Nevertheless, if it helps the development of agricultural research and business in the Midwest in general, then there are very good reasons to for neighboring states to support this in general - and this has occurred. 
A second obvious barrier to regional collaboration is that we live in a relentlessly global marketplace for talent and capital. Globalization can make regional collaboration look like a weak and inefficient development strategy doomed to fail. The potential influence of this dynamic should never be ignored. For example, a state may invest tax revenue into the development of various bioscience companies to help them get started, grow and prosper. But for most investors in such companies, it's simply logical from a global business perspective that once they have proven their value, some larger international company will swoop in and buy them.

The third obvious barrier to regional collaboration is distance. Once the physical distance between any two scientists requires much more than an hour or so to traverse, then if may be just as easy to collaborate with someone hundreds or thousands of miles away as the regional colleague. That is, once the response cost of collaborating with someone in the region grows beyond a rather low time/distance threshold, its regional advantage may be gone. Then the relentless forces of globalization can take hold. Remember, scientists and innovators don't collaborate to be nice; they do it to solve problems efficiently and effectively.

\section{Incentives for Regional} Collaboration

What are some potential incentives for regional collaboration? Perhaps the most obvious one is the presence of world class scientific talent in the region itself. This is the edge that the Boston area has in general has with its many world class private universities, as do regions such as the Bay Area in California, and the Research Triangle in North Carolina. We have no such concentrations in the Midwest and instead our great research universities are separated by relatively large distances. These concentrations of talent obviously enable regional collaboration. Fortunately, there are several strategies to overcome the talent concentration challenge. The most obvious one is to simply take advantage of recognized regional strengths. Hence, the recognition in recent years of the large number of companies the make up the so-called animal health corridor in Missouri and Kansas.

Explicit incentives for regional collaboration include the provision of funds to support it and opportunities that require it. Thus, many states are investing substantial funds in various bioenergy initiatives and the biosciences in general through a host of financial incentives. How well these kinds of efforts will work in the long run is yet to be determined. Less expensive incentives can be provided as well. For example, universities could provide modest amounts of direct support for the collaborative efforts of their faculty to work with faculty from neighboring universities. Will that matter in the long run? Again, that remains to be seen. However, I've always been impressed by the large impact that can often be achieved from very small investments that essentially prime the pump to get collaborations going. Supportive administrative policies can also help, as can simply having a history of successful collaboration. Success breeds success. However, in the end it's probably the 
case that nothing insures regional collaboration better than a problem that truly requires a regional solution.

\section{Concluding Comments}

It's important to recognize that regional collaboration and competition can go hand in hand. How did Wichita, Kansas, become an international force in the commercial aviation industry? Both collaboration, including scientific and technological, and competition were part of the story. And the fundamental story behind the Wichita aircraft industry doesn't differ all that much from the development of Silicon Valley in the Bay Area. The point is - regional collaborations, especially among scientists and businessmen have spawned many extraordinary success stories even in the very recent past, and even in a globalized world. 